\title{
XI Convegno della Società Canadese per gli Studi di Italianistica
}

L'XI convegno della Società Canadese per gli Studi di Italianistica ha avuto luogo a Vancouver presso l'università della Colombia Britannica, tra il 30 maggio e il 3 giugno 1983, nell'ambito dell'incontro annuale delle Società Accademiche Canadesi. Sebbene il programma, suddiviso nelle abituali quattro sezioni, si presentasse ricco ed invitante, date le enormi distanze e gli alti costi di viaggio, si pensava che il pubblico sarebbe stato scarso. Invece è stato proprio il contrario: i partecipanti sono stati numerosi ed interessati.

La sezione dedicata al Medioevo, diretta da Pamela Stewart (McGill University), si presentava particolarmente attraente sia per l'alto numero di interventi sia per la varietà dei temi trattati. In "Dante's Divine Komos: Aspects of Carnival in the Commedia," Richard A. Cavell (University of Toronto), si è chiesto quali fossero gli aspetti fondamentali per cui il poema dantesco potesse essere veramente considerato una "commedia." Attraverso un'accurata analisi degli elementi carnevaleschi presenti nell'opera, ed applicando il modello del dialogismo sviluppato da Mikhail Bakhtin alla struttura di tutta l'opera, ha concluso che il comico implica un tipo di opposizione non esclusivo, e che, data la serietà ascritta alla commedia dalla cultura medioevale, il termine "commedia" si applica perfettamente alla visione alta ed universale del poeta. Nella sua lineare e convincente presentazione Antonio D'Andrea (McGill University), in "Dante e la memoria: la Vita nuova" ha sottolineato che lo scarto fra prosa e poesia nella Vita nuova sembra mettere in questione la dichiarazione iniziale dell'autore, e cioè la sua intenzione di copiare, $d^{\prime}$ "assemplare" le parole che egli trova scritte nel libro della sua memoria, "e se non tutte, almeno la loro sentenzia." Tuttavia per D'Andrea il nodo della questione è in una nozione della memoria che - nel suo duplice significato di conservazione e rievocazione del passato - ha il suo modello nell'allegoria figurale propria dell'esegesi biblica. Amilcare A. Iannucci (University of Toronto), in "The Harrowing of Dante from upper 
Hell (Inferno VIII. 67 - IX. 105)" in maniera drammatica ha mostrato come il punto focale del IV canto dell'Inferno non è tanto la discesa di Cristo nell'inferno, quanto la tragedia dei pagani virtuosi. Dante, però, conscio delle possibilità drammatiche del tema, ne rievoca le tradizionali immagini agonistiche nei canti VIII e IX dell'Inferno combinando elementi cristiani e pagani, e lo recupera di fronte alle mura della città di Dite. In "Oriental Sources of novella VIII, giornata VIII, of Boccaccio's Decameron," Gabriele Erasmi (McMaster University), rendendo vivida la vis comica del Boccaccio, ha mostrato come la novella non ha precedenti né classici né medievali. Esaminata dal punto di vista di psicologia sociale, la situazione in essa presentata non si accorda in nessun modo con la moralità tradizionale occidentale. Si presume quindi che sia di derivazione orientale (tramite il mondo arabo) in quanto essa si accorda con gli atteggiamenti riguardanti l'adulterio che si ritrovano nel Kama Sutra. Pertanto il Boccaccio avrebbe occidentalizzato il racconto con l'inserzione di elementi psicologici borghesi. Dennis McAuliffe (University of Toronto), nel discutere "Some unusual signs of the Sacrament of Baptism in Dante's Comedy," ha sostenuto che il modo in cui Dante si è servito del simbolismo tipologico dei sacramenti è centrale alla ermeneutica della Divina Commedia. L'inizio del canto XXV del Paradiso è indice della

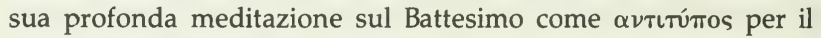
тú base del metodo che viene spiegato nella lettera a Can Grande della Scala. In tal modo passi tradizionalmente ritenuti di difficile interpretazione - quale l'episodio biografico intromesso nel canto dei simoniaci (Inf. XIX) -, possono godere di nuove possibilità interpretative. In "Le origini dei drammi della Passione" Salvatore Bancheri (University of Toronto) ha chiaramente mostrato come con la scoperta della Passione di Montecassino sia venuta a cadere la tesi di Chambers e di Young che ricercavano le origini dei drammi della Passione nel canto dialogato del Passio e del Planctus. Per lui, in accordo con lo Sticca, l'interesse per queste rappresentazioni scaturisce da un nuovo atteggiamento verso la figura di Cristo e da un rinnovato interesse per le sue sofferenze. Queste idee ebbero come nucleo embrionale l'abbazia di Montecassino che fu l'ambiente ideale per la suddetta opera, la quale deve essere considerata come il prototipo dei drammi della Passione. Paul Colilli (University of Toronto), in "Petrach's Africa," partendo da un punto di vista semiologico, si è proposto di mettere in evidenza come l'Africa del Petrarca sia un testo autosufficiente che racchiude un referente immanente. Ha esaminato le implicazioni teologiche di tale autosufficienza facendo particolare riferimento ai 
passi autoreferenziali, per poi concludere che il Petrarca voleva creare con l'Africa una versione poetica dell'Età dello Spirito Santo quale concepita da Rupert di Deutz e da Gioacchino da Fiore.

La sezione dedicata al Rinascimento e al Barocco, diretta da Walter Temelini (University of Windsor), è stata piuttosto scarsa a causa della mancata partecipazione di due relatori; tuttavia l'interesse creato da quelli che vi hanno partecipato è stato notevole. Michael Lettieri (University of Toronto), in "L'Orazia di Pietro Aretino: tra bembismo e antibembismo," ha sottolineato come per la critica l'immagine piú vulgata di Pietro Aretino è quella dello scrittore che in teoria ed in pratica si pone in netta opposizione al normativismo bembiano. Ha aggiunto che è stato anche giustamente notato come alcune opere dell'Aretino si collochino negli schemi di quel pedantismo che egli stesso critica con tanto vigore. Ed ha concluso che un'ulteriore prova che l'Aretino sia meno monolitico di quanto si pensasse è l'Orazia, la quale risulta dominata, sebbene non pedissequamente, dall'adesione alla prassi desumibile dalle Prose di Pietro Bembo. In "Il concetto di poesia nelle opere del Gravina," Domenico Pietropaolo (University of Toronto), ha finemente analizzato la nozione di poesia di Gianvincenzo Gravina nelle sue componenti concettuali, studiate nella prospettiva delle tradizioni culturali da cui esse derivano e nel contesto della sua teoria generale della letteratura. Ha concluso che l'originalità del classicismo graviniano consiste principalmente nell'importazione dell'idea di giurisprudenza nella poesia e nel trasferimento del poeta nel ruolo del giureconsulto, educatore degli uomini secondo l'ordine assiologico del mondo civile, di cui egli ha una comprensione superiore. Filippo Salvatore (Champlain College), in "Il tacitismo nel pensiero politico della Controriforma: Tacito come maschera del machiavellismo" ha sostenuto che il tacitismo non è una semplice maschera del machiavellismo che riduce la religione a puro instrumentum regni come ha sottolineato Toffanin o la critica laica. Per il Salvatore, durante la Controriforma, il culto di Tacito, che sostituisce quello di Livio, è strettamente connesso a quello di Seneca. Il teatro orroroso di Seneca trova riscontro nella visione della storia vista come "tragica scena." Il contributo precipuo dei tacitisti non è su un piano di elaborazione teoretica, ma di morale e di stile.

Estremamente ricca e varia la sezione che Rocco Capozzi (University of Toronto), ha coordinato sul periodo moderno e contemporaneo. Massimo Verdicchio (University of Alberta), in "Aesthetic and Ideology in Croce's Estetica" partendo dal concetto che la lunga meditazione crociana sulla filosofia dell'arte è sintomatica di una problematica che si manifesta a livello testuale nell'ambiguità 
dei termini che definiscono il fatto estetico, ha mostrato che anche se Croce sembra favorire una teoria dell'arte come simbolo, la tesi non è comprovata in quei luoghi dove egli riflette sulla natura dell'arte. Ha concluso che le ragioni della discrepanza sono nel fatto che ciò che per Croce è l'artistico, nell'estetica idealistica tedesca a cui egli si rifà, viene definito inartistico. Anna Urbancic (University of Toronto), in "Tu se' donna: il rapporto letterario fra Niccolò Tommaseo e George Sand" dopo aver notato che la recezione dei romanzi della Sand era stata sempre mista, fa il caso particolare di Niccolò Tommaseo. Per lui dapprima essi erano una "robaccia," poi vi trova un "giusto equilibrio tra sensualità e misticismo" per cui ammira la Sand come una donna ideale, ma non veramente perché "dotta." La Urbancic conclude con un'analisi della poesia La donna. A Giorgio Sand (1835) in cui vengono messi in evidenza alcuni dei motivi dell'ammirazione del Tommaseo per la scrittrice francese. Particolarmente viva e interessante la presentazione (con diapositive) de "I quadri di Luigi Pirandello" di Antonio Alessio (McMaster University), il quale ha preferito definire questo suo intervento "storia di un'indagine" piú che relazione vera e propria, la quale presuppone sempre delle conclusioni e delle proposte. Alessio, nel presentare brevemente la trentina di quadri di Luigi Pirandello da lui finora ritrovati, e rifacendosi alle sue recenti e correnti indagini sui rapporti tra pittura e opera letteraria, ha sottolineato, al di là del valore pittorico vero e proprio dei quadri stessi, la notevole importanza documentaria di questa produzione, anche in relazione all'idea estetica pirandelliana. In "Il rapporto tra il fotografo e la macchina fotografica in Si gira . . . e Blow up" Giuliana Sanguinetti Katz (University of Toronto), ha esaminato il rapporto che l'operatore di Si gira . . . e il fotografo di Blow up hanno sia con la loro macchina fotografica sia con il mondo esterno ed interno con cui vengono a contatto tramite appunto la loro macchina. Per la Katz in entrambe le opere il protagonista si serve della macchina per controllare il mondo esterno e dar sfogo ai suoi istinti proibiti. In entrambi i casi il protagonista, messo in contatto dalla macchina con le sue fantasie edipiche, non riesce a risolvere i suoi conflitti e perde il suo senso di identità. Franco Ricci (University of Ottawa), in "The loss of self and silence in Gli amori difficili by Italo Calvino," ha acutamente indagato lo sdoppiamento del personaggio e la ricerca di una via di uscita dal "labirinto" socio-esistenziale. Per lui in questi racconti si nota la tematica dominante dell'autore che va da una problematica sociopolitica verso quella ermetica ed esistenziale (verso "il silenzio" che si noterà nei suoi racconti degli anni sessanta). Ha concluso che Gli amori difficili devono essere considerati come una 
fase intermediaria nello sviluppo della narrativa di Italo Calvino. In "Literature as polemics in Maraini's Donna in guerra and Memorie di una ladra," Vera Golini (University of Waterloo), ha messo in evidenza che sin dalla fase iniziale della sua attività letteraria, la tematica base di Dacia Maraini è stata l'inquietudine della donna oggetto presa dalla disperazione della vita abbietta nelle grandi città. Ha poi analizzato in particolare Memorie d'una ladra e Donna in guerra per concludere che in esse la Maraini ci dà il meglio della sua produzione letteraria attraverso la figura di due donne la cui presa di coscienza e conseguente rivolta sono una vera e propria denuncia sociale della condizione della donna nella società odierna. Giuseppe Faustini (Skidmore College), in "Dorpelli/ Pirandello as a literary reviewer" esamina una scelta ragionata delle numerose recensioni pirandelliane firmate con l'anagramma Giulian Dorpelli e che fecero parte dal 1896 al 1901 della rubrica "Fra libri vecchi e nuovi" del settimanale romano di Federico Garlanda, Rassegna settimanale universale (poi Minerva). La recensione pirandelliana che ha suscitato maggior polemica è la stroncatura in parte, della quarta edizione delle Myricae (1897) di Giovanni Pascoli. Altre recensioni esaminate sono: La sfinge (Luigi Capuana), Giacomo l'idealista (Emilio De Marchi), L'amuleto (Neera), Nelle tenebre (Giuseppe Baffico), e Poesie scelte di Antonio Fogazzaro.

Ricca e nutrita anche la sezione dedicata alla linguistica applicata ed alla pedagogia, diretta da Marina Frescura (York University). La vivida presentazione di Raffaella Maiguashca (York University) "Insegnamento del lessico: il rapporto di antonimia" fa parte di un lavoro di ricerca in corso che ha come scopo la formulazione di una metodologia per l'insegnamento del lessico in cui vengono applicati i principi e i concetti delle teorie semantiche moderne. Il materiale didattico presentato, consistente di una serie di esercizi sui cosidetti "antonimi" o "contrari," è un esempio illustrativo di questa nuova metodologia. Partendo dalle analisi del rapporto di antonimia fatte da linguisti come Lyons, Leech, Coseriu, ecc., si propone al discente una classificazione degli antonimi, cioè dei differenti tipi di opposizione semantica, e lo si porta gradualmente a "lavorare" sulle parole mediante tecniche pedagogiche appropriate. In questo modo si spera di contribuire a rendere lo studio del vocabolario piú rigoroso, piú sistematico, piú efficace. Vittorina Cecchetto (McMaster University), in "Osservazioni sull'uso dell'articolo con i nomi geografici" ha mostrato come l'uso corretto dell'articolo con i nomi geografici e piú specificamente con le isole, costituisca una grossa difficoltà nell'insegnamento dell'italiano a stranieri. Regole date da grammatiche di tipo tradizionale o anche moderne non bastano a spiegare le 
numerose eccezioni. Da un'analisi di riviste contemporanee, romanzi di avventure, o l'Atlante geografico De Agostini è possibile ricavare regole quali: 1 . l'articolo si adopera sempre: a) quando il nome è al plurale, b) al singolare con specifici nomi di isole; 2 . si omette in tutti gli altri casi. Jana Vizmuller-Zocco (University of Toronto), in "Chiedere 'scusa' in inglese e in italiano" ha esaminato questa particolare situazione sociale nelle due lingue ed ha descritto questo atto utilizzando il concetto di "pragmena". Il pragmena è quella situazione extralinguistica che richiede il proferimento di un enunciato. Per quanto riguarda chiedere scusa, ha concluso, pragmeni quali "infrangere norme sociali" 0 "invadere lo spazio dell'interlocutore" comportano diverse risposte corrispondenti in inglese e in italiano. Carlo Fonda (Concordia University), in "An analysis of some phonetic errors in the speech of near-bilinguals whose mother tongue is Italian," ha messo in evidenza che l'uso generalizzato delle consonanti occlusive con realizzazione esplosiva è una caratteristica notevole del cosidetto "accento" degli italofoni che apprendono l'inglese. Questa mancata distinzione fra gli allofoni delle occlusive dell'inglese provoca delle alterazioni non soltanto nei tratti fonetici dei fonemi in questione, ma pure della struttura fonologica dei vocaboli stessi. Tale comportamento linguistico ci fa supporre che in questi casi l'apprendente operi un trasferimento negativo (TN) di restrizioni fonologiche soggiacenti, piuttosto che il trasferimento dei singoli fonemi. Esperimenti svolti con locutori del mandarino sembrano confermare la nostra ipotesi, in quanto pure questi parlanti tendono a modificare la struttura fonologica delle parole inglesi di modo che questa coincida con il sistema sillabico del mandarino. Lo scopo della comunicazione di Nicoletta Villa (York University) "La pragmatica e l'insegnamento delle lingue straniere," è stato quello di illustrare le carenze assiomatiche che l'approccio comunicativo presenta. Tali carenze possono infirmare completamente l'approccio in quanto non offrono la possibilità di giudicare della verità o falsità (accettabilità o no) degli enunciati prodotti. La Villa ha concluso con la presentazione di due tipi di proposte: a) regole che spiegano l'uso situazionale degli atti linguistici, e b) apparati cognitivi che organizzano il discorso dal punto di vista della teoria dell'enunciazione. Per Diego L. Bastianutti e James K. MacDonald (Queen's University) "Queen's VINCI: an integrated Programme of Italian Language," la concezione, disegno e implementazione del programma $Q^{\prime}$ VINCI per la lingua italiana rispecchia un'architettura e strategia che offrono al professore utente un elevato grado di flessibilità nella creazione di materiale didattico individuale, l'elaborazione dei punteggi e l'analisi dei quesiti, l'analisi e 
lo spoglio elettronico delle schede di risposta del discente (cioè il "feedback" intelligente), e la somministrazione diretta di prove di controllo. Il programma $\mathrm{Q}^{\prime} \mathrm{VINCI}$ non è abbinato a un libro di testo particolare. Si basa bensí sul lessico di frequenza stabilito da Sciarone suddiviso in una serie di "Data Banks" le cui voci sono individualmente codificate per la loro funzione morfologica, sintattica, ecc. Il materiale didattico viene creato dal professore presso una unità d'ingresso (computer terminal) grazie a uno schema codificato che gli permette di ordinare la generazione dinamica di esercizi direttamente all'unità centrale (mainframe computer), e indi proposti al discente presso una unità d'uscita (computer terminal a schermo). Data la vasta capacità delle "Data Banks" codificate e del "Random Access" del Q'VINCI, ogni professore è in grado di creare un programma individualissimo che risponda al suo corso di studio particolare. $Q^{\prime} \mathrm{VINCI}$ è il nome depositato del programma.

È terminato cosí l' XI convegno della Società Canadese per gli Studi di Italianistica, ricco e fruttuoso sia per i risultati a cui si è pervenuti, sia per i numerosi e pertinenti interventi del pubblico che hanno illuminato ancor piú la materia trattata, sia per le nuove aperture ed orizzonti che si sono delineati. L'appuntamento per la prossima primavera è a Guelph.

LEONARD G. SBROCCHI

University of Ottawa 